\title{
La biomecánica del aparato locomotor en España
}

\section{A. VILADOT PERICEE}

Profesor titular de la Universidad Autónoma de Barcelona Ex-Presidente de la Sociedad Ibérica de Biomecánica

La aplicación de los principios de la mecánica a la fisiología del aparato locomotor ha estado latente en la biología, la física y el arte, por ello, al hablar de los antecedentes de la biomecánica deberíamos remontarnos a Hipócrates o Arquímedes, pasando por Leonardo da Vinci. Es curioso que en el Siglo XVI, para tener categoría social, los artistas debían hacerse científicos y quizá ésta puede ser la explicación de los geniales hallazgos antropológicos de un Leonardo o de un Durero. Por otra parte, desde el punto de vista matemático no podemos olvidarnos de la personalidad de Newton, cuyos principios siguen siendo vigentes.

Al referirnos a la Biomecánica en España, consideraremos de una parte, los antecedentes, el estado actual de la misma que, aunque hemos procurado hacerlo lo más completa posible, forzosamente tendrá omisiones, que lamentamos y procuraremos subsanar. Finalmente, para que el pasado tenga valor debemos pensar en el futuro, más teniendo en cuenta que ya en estos últimos años la biomecánica del aparato locomotor se ha mostrado imprescindible para el progreso de la cirugía ortopédica y traumatología.

\section{ANTECEDENTES}

Al situarnos en la España del Siglo XVI hemos de pensar que en aquel entonces nuestro Imperio se extendía por los Países Bajos, Italia y el Nuevo Continente. Así aunque el padre de la antomía moderna Andrea VESALIO (1514-1564), con su obra magistral «Humanis Corporis Fabrica», naciera en Bruselas, entonces los Países Bajos pertenecían a España, y él fué protegido por nuestros Reyes, Carlos I y Felipe II, que lo nombraron su médico personal.

Felipe III, mandó tropas españolas al reino de Nápoles. Un soldado de las mismas, fué don Miguel ALFONSO, casado con una italiana Laura BORELLI. De este matrimonio nació en gran Giovanni ALFONSO BORELLI (1608-1679), que escribió el primer tratado de biomecánica del aparato locomotor y que bien puede considerarse como el fundador de la biomecánica.

En el prólogo de su libro escribe: «Mi objetivo es describir la difícil fisiología del movimiento de los animales, es cierto que se ha hecho en muchas ocasiones por los antiguos y por los modernos, pero ninguno que yo sepa, ha estudiado los numerosos problemas importantes e interesantes de conocer que se puedan discutir, ni ha sabido o querido confirmar sus estudios con demostraciones mecánicas. Yo he iniciado mi obra porque esta parte de la física ilustrada y reforzada por demostraciones matemáticas puede incluirse dentro de las Ciencias físicas, matemáticas y la astronomía». Es difícil dar una definición mejor de la Biomecánica.

Por otra parte no podemos olvidar el Siglo en que nos encontramos y dentro de la misma obra se ve el carácter metafísico que ALFONSO BORELLI sabía dar a sus investigaciones, cuando afirma «Los cirujanos anatomistas y los filósofos no se preocupan de comprender aquel lenguaje con el cual el Creador de la naturaleza suele expresar sus propios conceptos en éste código sensible. El divino Platón, cuando uno le preguntaba qué cosa hacía la divinidad, respondía: «Dios hace el geometra», puesto que El hace geometría. Esta idea bien conocida por hombres doctos se adapta a nuestros propósitos».

La obra va ilustrada con una serie de esquemas interesantísimos (Fig. 1). Podemos que aún en el momento actual es útil la lectura de esta obra.

Varios autores se interesaron por el estudio de la Biomecánica del movimiento en el siglo pasado, WOLF, CULMAN, ROUX describió sus famosas Leyes, FICK que escribió un excelente tratado de medicina física, HUETER, etc...

Es curioso hacer constatar que el gran empuje que se dió al estudio de la biomecánica a principios de siglo, coincidió con la revolución futurista que culminó en el manifiesto de MARINETTI en 1909. Esta, entre otras cosas, destacó la gran influencia de los progresos de la ciencia y su influencia en la sociedad y se preocupó de plasmar gráficamente el movimiento, tanto en la pintura como en la literatura. Las letras ya no se limitaban a formar palabras para exprsar conceptos, sino que cambiaban de posición. Esta obsesión para la representación gráfica de la motilidad se completó con dos descubrimientos científicos importantes, primero la fotografía y después, la cinematografía. MAREY fue el genial investigador francés que empleó estos métodos para el estudio de la motilidad de los animales y del hombre (Fig. 2), y de allí surgieron los primitivos estudios sobre la marcha.

Poco después se publicaron obras trascendentales para el conocimiento de la biomecánica del aparato locomotor: el libro de STEINDLER «Kynesiology», editado en 


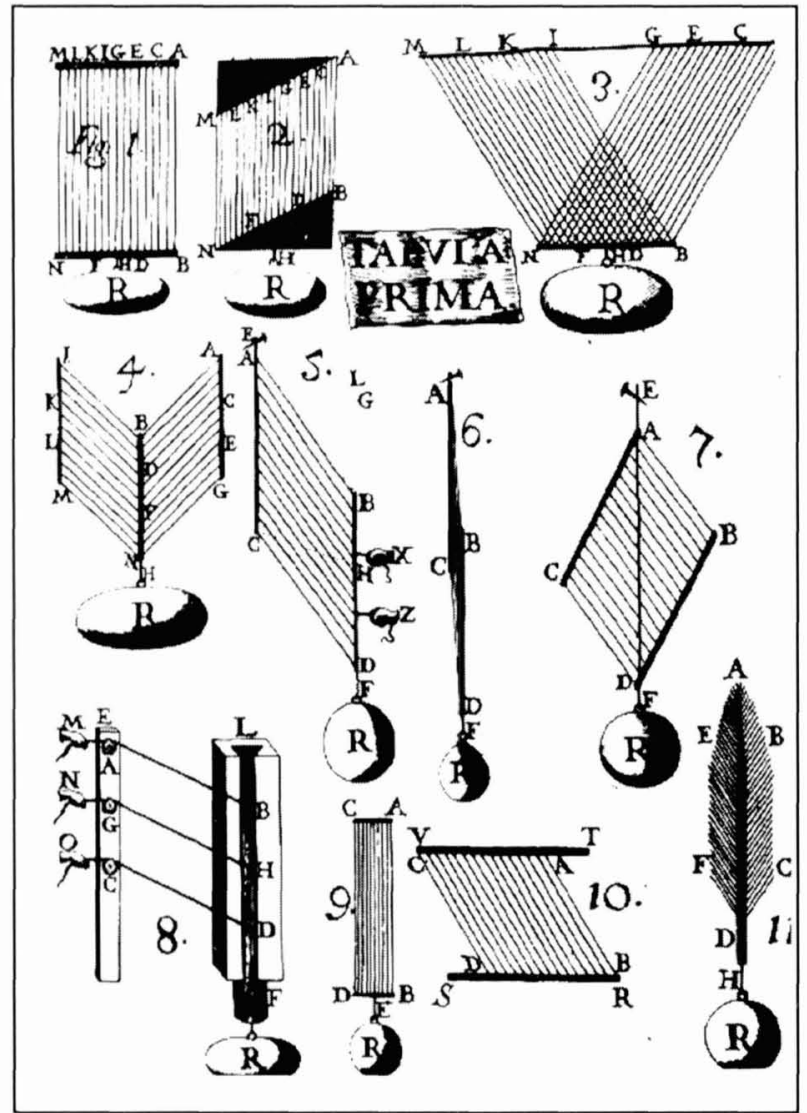

Fig. 1. Grabado del libro de «Motu Animalium» de Giovanni ALFONSO BORELLI.

1955, y el artículo aparecido en el Journal of Bone and Joint Surgery, «The major determinants in normal and pathological gait», de SAUNDERS y colaboradores.

En el Hospital de San Juan de Dios de París, Charles DUCROQUET continuado después por sus hijos, Robert, Jean y Pierre, estudió científicamente la marcha con la ayuda de la cinematografía e ideó su famoso pasillo de espejos que permitía una visualización tridimensional del cuerpo en movimiento.

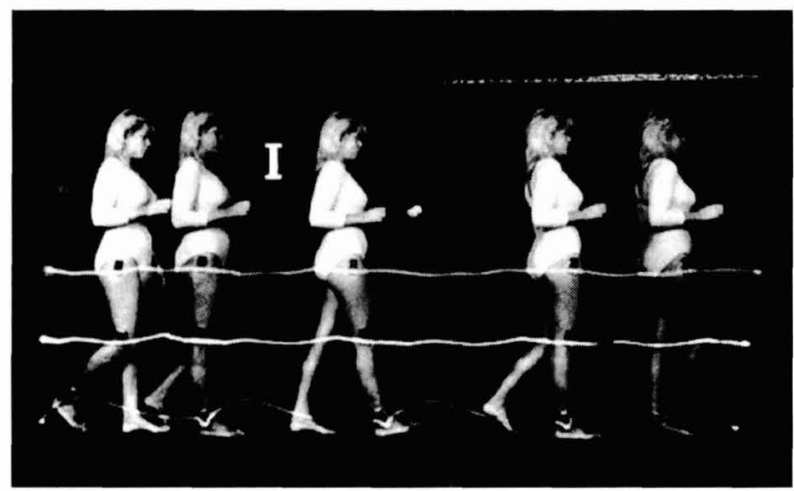

Fig. 2. Estudio fotográfico de la marcha.

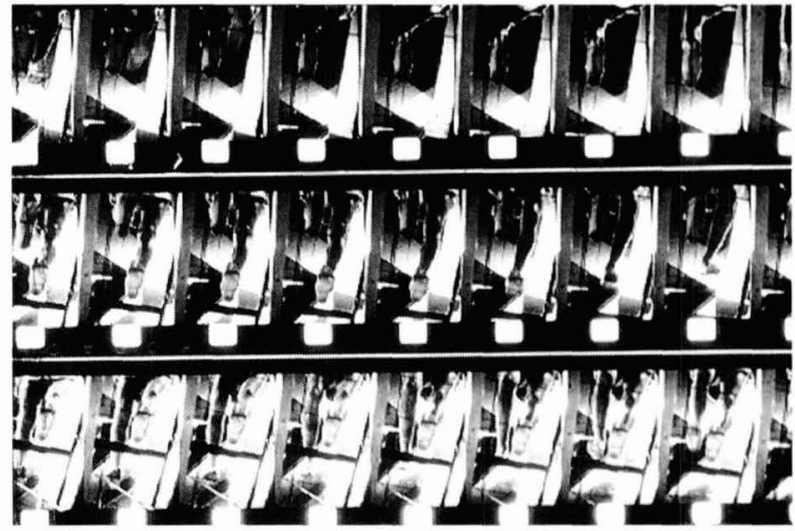

Fig. 3. Cinematografia de la cadencia del apoyo plantar en el pasiIlo de espejos del Hospital de San Rafael.

En el Hospital de San Raael instalamos en 1965 este pasillo de espejos con el cual hicimos estudios cinemáticos de la marcha normal y patológica (Fig. 3). Fruto de los mismos fueron diversas publicaciones y Tesis Doctorales, entre ellas la de Antonio VILADOT, Jr., en colaboración con el Laboratorio de Marcha de MANN en San Francisco, primera sobre el tema en España.

Junto con el pasillo de espejos y basados en una sugerencia del señor Pablo VILATO, sobrino de Picasso, presentamos un estudio fotográfico de la huella plantar y así nació el fotopodograma, con sus derivados, el fotoestatograma y el radio-fotopodograma.

\section{ESTADO ACTUAL}

En el momento actual las líneas de investigación de la biomecánica en España se realizan fundamentalmente a través de los Institutos Nacionales de Educación Física (I.N.E.F.), de las Facultades de Medicina, las Universidades Politécnicas, algunos Hospitales y determinados centros privados. Fruto de las mismas son la gran cantidad de Tesis Doctorales que han aparecido en estos últimos años y que demuestran que a pesar de la tradicional penuria económica que tenemos en nuestro país para la investigación, ésta sea respetable. Estimulando esta labor está la Sociedad Ibérica de Biomecánica.

\section{I.N.E.F.}

Estos han sido el primer centro oficial en que se ha estudiado la Biomecánica con carácter obligatorio en las asignaturas de «Biomecánica del Movimiento Humano» y «Biomecánica de las técnicas deportivas».

Esta oficialidad ha hecho que, junto con la docencia se cultive la investigación y así, la I.N.E.F. en Cataluña (el Director J. SACHA), en Barcelona ha trabajado sobre el tema Eugenio ESPARZA y muy especialmente en Lérida, los Profesores BIOSCA y GARCIA FOJEDA, con rigor científico investigan sobre el gesto deportivo (Fig. 4). Destacan también los estudios en biomecánica de las Universidades de Granada y del País Vasco. 


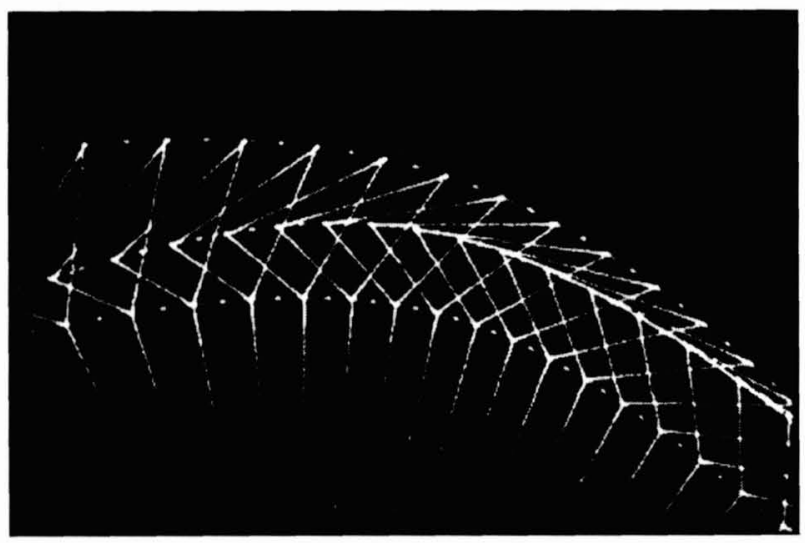

Fig. 4. Estudio cinemático del movimiento en el futbolista (I.N.E.F. Lérida Dr. J. BIOSCA).

\section{FACULTAD DE MEDICINA}

La investigación biomecánica destaca en varias Universidades. En Madrid, en la Universidad Complutense, en el Departamento de Ciencias Morfológicas (Prof. J. JIMENEZ COLLADO), bajo la dirección del Prof. Pedro GUILLEN, se imparte un Master en Biomecánica del Aparato Lomotor en íntima colaboración con la Escuela de Medicina del Deporte.

En el Departamento de Ciencias Morfológicas del Prof. J. PUERTA FONOLLA, se dan, desde 1981, Cursos de Doctorado de Anatomía Funcional y Biomecánica del Aparato Locomotor. Más recientemente, un Curso de Master sobre «Biomecánica y Cirugía del Pie». Es el entusiasmo del Prof. Fernando LLANOS el alma de estas enseñanzas. Resultado de las mismas ha sido la publicación del primer Tratado de Biomecánica publicado en España (Fig. 5).

En esta misma Universidad ya hace unos años Fernando LLANOS y Mariano NUÑEZ SAMPER trabajaron intensamente sobre la Electromiografía en el pie normal y patológico que fué tema de sus Tesis Doctorales.

En la Universidad de Alcalá de Henares, el Departamento del Prof. L. GOMEZ PELLICO ha organizado un excelente laboratorio de Biomecánica. En él tienen plataformas cinéticas, cámaras para estudios cinemáticos, tensimetría, radiología, electomiografía, etc..., todo lo cual se ha traducido en diversos trabajos de alto valor científico y Tesis Doctorales que figuran en los apartados correspondientes.

De forma semejante a Madrid, en Barcelona han sido los Departamentos de Ciencias Morfológicas de nuestras Facultades de Medicina, los que han presentado mayor dedicación al tema. En la Universidad Autónoma el Prof. DOMENECH ha estudiado las propiedades físicas de los tejidos y la anatomía funcional del aparato locomotor. El nivel de su labor se demuestra al haberle sido concedido en este último año, el Premio E.B. KAPLAN, que es el más importante galardón que concede la Sociedad Americana de la Cirugía de la Mano.

En la Universidad Central, el Prof. Domingo RUANO, uno de los científicos de nuestro país que más

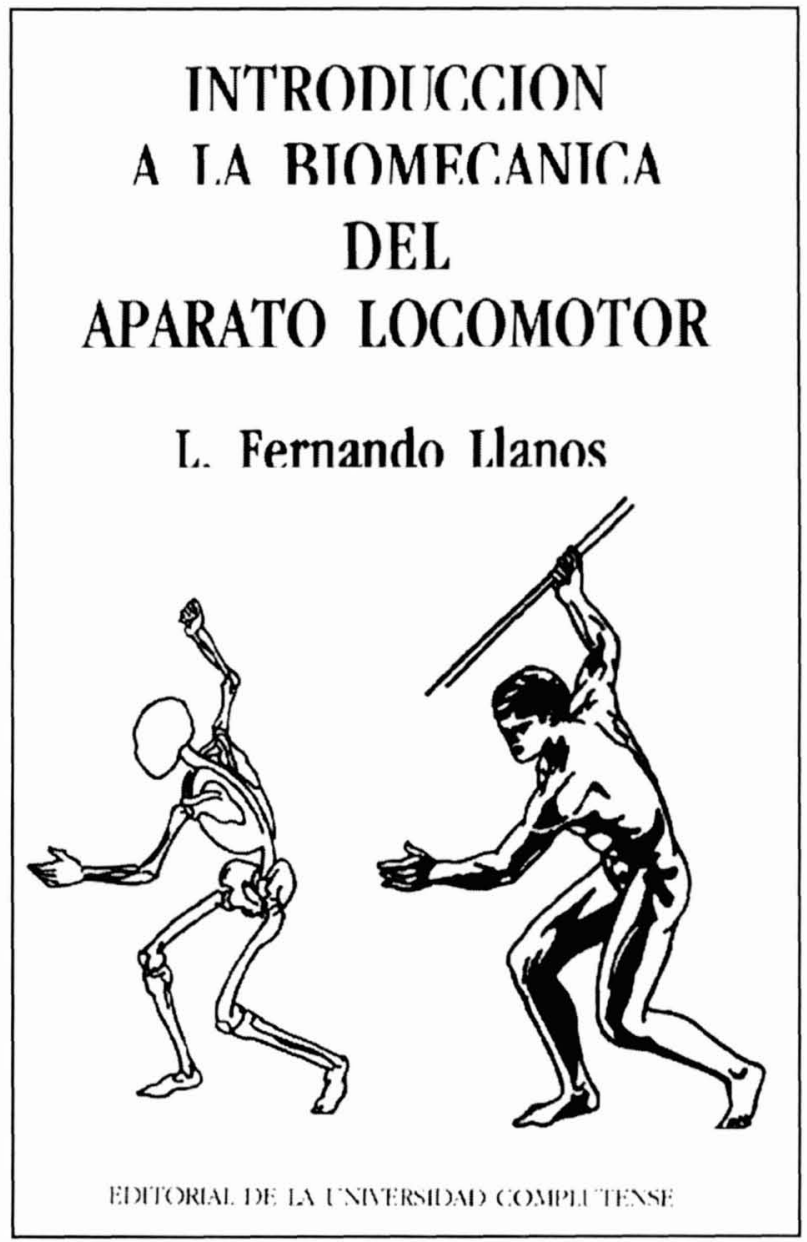

Fig. 5. Primer tratado sobre biomecánica del aparato locomotor publicado en España.

ha impulsado los estudios de embriología y anatomía de sus diversas vertientes, no se ha limitado a los aspectos teóricos, sino que ha impulsado sus aplicaciones prácticas. Buena prueba de ello es la creación de la Escuela de Medicina de la Educación Física y del Deporte.

Tiene un excelente laboratorio (Fig. 6), dirigido por el Prof. MORGENSTERN, con un «peak performance» para el estudio de la marcha. En colaboración con la I.N.E.F. ha hecho estudios cinesiológicos sobre diversos deportes y junto con la Escuela de Ingenieros está estudiando la Biomecánica de los Tendones y Ligamentos. En el mismo departamento se imparte, con regularidad, Cursos de Post Grado en Biomecánica y de Formación de Técnicos Ortopédicos.

En la Facultad de Medicina de la Universidad Autónoma y en el marco de la Cátedra del Prof. A. NAVARRO se han hecho valiosos estudios sobre la Biomecánica de la Rodilla. En su Servicio del Hospital del Valle de Hebrón, el Dr. PLAJA ha investigado la marcha humana, especialmente por métodos electrogoniométricos y electromiográficos. En el Hospital Germans Trías, ROCA BURNIOL y en colaboración con la Universidad Politécnica se ha dedicado a la mecánica de la columna vertebral. 


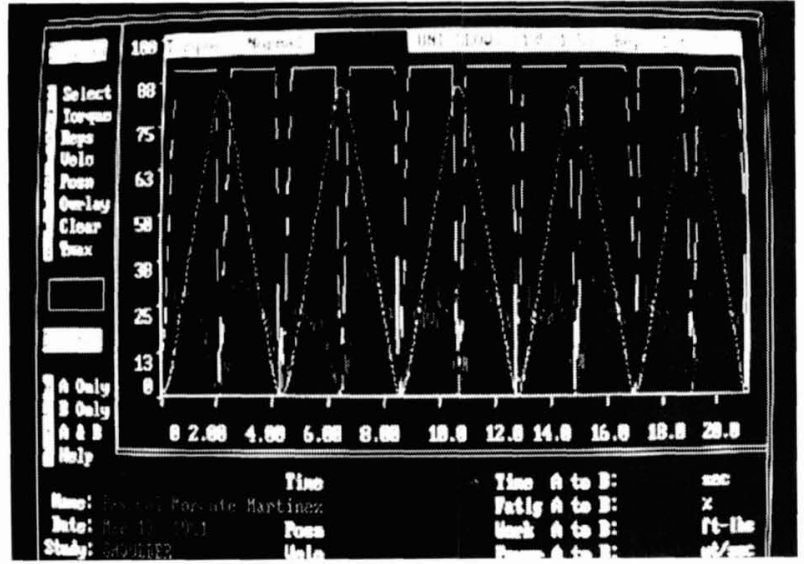

Fig. 6. Biomecánica del hombro (Prof. L. GOMEZ PELLICO. Universidad de Alcalá de Henares).

También dentro de la misma Cátedra, en el Hospital de San Rafael, hemos organizado desde 1979, catorce Cursos de Biomecánica. La línea de investigación primero con el pasillo de espejos, se ha centrado en el estudio de la marcha normal y patológica.

Evidentemente, con Barcelona y Madrid no se acaba la investigación universitaria. En la Universidad de Navarra, y dentro del Departamento del Prof. CANADELL, trabaja últimamente con gran entusiasmo el Prof. J. FARRIOL. Debemos citar también y aún conscientes de muchos olvidos, las Universidades de Córdoba, (Prof. J. PEINADO), Valencia, (Prof. V. SMITH AGREDA), Valladolid, (Prof. S. SANCHEZ MARTIN), Zaragoza, (Prof. I. SERAL IÑIGO), Cádiz, (Prof. J.A. COPANO ABAD).

\section{UNIVERSIDADES POLITÉCNICAS}

La Universidad Politécnica de Catalunya, aparte de colaborar con entusiasmo a través de los Profesores PLANELL y VAZQUEZ, en las labores de docencia y de investigación que se dan en diversas Universidades, hacen una valiosa investigación propia, tanto desde el punto de vista de la Biomecánica del Aparato Locomotor (Trabajo sobre Rodilla, Columna Vertebral, Tendones, etc...), como sobre instrumental quirúrgico, en la actualidad en colaboración con el Dr. FERNANDEZ-FAIREN se está trabajando sobre biomateriales.

Lugar muy destacado merece la Universidad Politécnica de Valencia, el pionero de los estudios de la misma, fué el Prof. Justo NIETO, uno de los primeros Presidentes de nuestra Sociedad. Dentro de su Departamento fundó un laboratorio que después se ha ido ampliando con el magnífico Instituto de Biomecánica de Valencia, en la actualidad bajo la dirección del Prof. Pedro VERA. Ha creado una tecnología propia. Diversos Centros españoles se han beneficiado con el uso de su excelente pasillo para el estudio cinético de la marcha.

En la actualidad y con un valioso equipo tiene tres líneas de investigación: Biomecánica Médica,
Biomecánica Ocupacional y Biomecánica Deportiva. Numerosos trabajos, Tesis Doctorales, Cursos y Congresos acreditan la calidad de su labor.

\section{INVESTIGACION NO UNIVERSITARIA}

Queremos resaltar la labor que en materia de investigación de Biomecánica en el Deporte se hace en el "Centro de Alto Rendimiento de Barcelona» (J.A. PRAT). Se estudia la cinesiología en diversos deportes. Su preparación se ha puesto en evidencia en los Juegos Olímpicos de Barcelona, donde se ha llevado a cabo un proyecto de investigación por el Dr. BALIUS y el Prof. ANGULO, cuyos resultados esperamos que sean realmente importantes. También en Cataluña podemos destacar el «Centro de Cirugía Experimental de la Mutua de Sabadellenca d'Assegurances», (su Director J. DOMINGO PECH, obtuvo un premio de la S.E.C.O.T. con una prótesis experimental de cadera). El Centre d'Estudis de Alt Rendiment Esportiu» (Dr. R. BALIUS JULI), Institut Catala de Traumatología i Medicina Esport (Dr. José María VILLARRUBIAS), «Institut de Ciencies Médiques i de l'Esport", (Dr. M. FERNANDEZ FAIREN), Hospital de la Esperanza (Biomecánica de la rodilla Dr. MARIN), Laboratorio de Biomecánica ASEPEYO (Prof. A. VILADOT y Dra. O. GALLIFA) que trabaja conjuntamente con el Servicio de Cirugía del Aparato Locomotor del Hospital de San Rafael.

En el Hospital Juan Canalejas de la Coruña, el Dr. Carlos PICHEL obtuvo la Beca del F.I.S. para estudios de Biomecánica, gracias a la misma realizó interesantes investigaciones sobre la rodilla normal y patológica. En la Mutua de Accidentes de Zaragoza, J. RAMOS y F. SOROLLA investigan con un pasillo cinético de marcha, también han presentado interesantes trabajos sobre la Morfología del Pié estudiado con T.A.C.

\section{INVESTIGADORES INDEPENDIENTES}

Las dificultades que existen en nuestro país para hacer investigación en entidades oficiales o privadas, ha motivado que diversos investigadores con auténtica vocación trabajaran de forma independiente. Aún a conciencia de muchos olvidos involuntarios, queremos citar los nombres de HERNANDEZ ROS, que en un principio trabajó en el Instituto Ramón y Cajal, y que después ha seguido estudiando lamarcha a través de robots, J.J. ZWART, por sus trabajos sobre Biomecánica de los tendones y Densimetría, J. MARTORELL, por sus estudios sobre el apoyo plantar y el calzado, CRESPO, también sobre Biomecánica del Pie y prótesis de astrágalo, etc...

\section{INSTRUMENT ACION EN CIRUGIA ORTOPÉDICA}

Uno de los aspectos de más directa utilidad de la Biomecánica del Aparato Locomotor es el tema de los implantes y del instrumental en cirugía ortopédica. En esto es notable la aportación española.

Como antecedentes y al mismo tiempo como prueba de lo mal que sabemos reconocer nuestros méritos, 


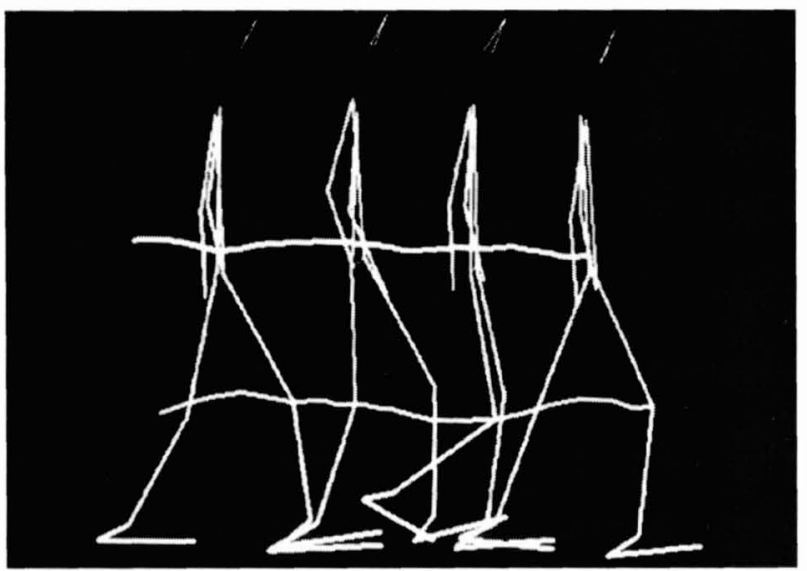

Fig. 7. Estudio de la marcha con el «Peak performance». (Profesores D. RUANO y F. MORGENSTERN.

debemos citar el fijador que en 1950 presentó J.L. MENDOZA (Fig. 7) y que constituye un claro precedente de tal divulgado y actual aparato de ILIZAROV. En Valencia, Alvaro LOPEZ en 1964 presentó su fijador vertebral que con mucha lógica enderezaba las curvaduras no estirando la columna sino por presión lateral y que tien el mismo fundamento del después tan conocido sistema de LUKE.

Dentro del capítulo de las prótesis de cadera y rodilla, hay que resaltar las investigaciones de F. AGUILAR, M. FERNANDEZ FAIREN, L. MUNUERA, J. PALACIOS, J.M. VILLARRUBIAS y el equipo de la Universidad Politécnica de Barcelona. Son notables los ensayos de sustitución del astrágalo por procesos plásticos y las agujas de inmovilización maleolar, presentadas por CRESPO-NECHES.

Una importante aportación ha sido el fijador externo de J. LAZO y J.M. CAÑADELL, que de forma dinámica facilita la compresión intermitente del callo óseo, tan importante para la consolidación de la fractura. Manuel L. CLAVEL, en el Hospital de la Seguridad Social de Murcia, está investigando sobre las aplicaciones de hidroxiapatita en cirugíaortopédica R. OROZCO a través de la Fundación Miller ha hecho importantes aportaciones a la osteosíntesis. Finalmente debemos citar nuestra ortesis y la de R. ALVAREZ para el tratamiento del pie plano.

\section{SOCIEDAD IBÉRICA DE BIOMECANICA}

El conjunto de labores tan dispersas a las que hemos aludido hacía necesaria una agrupación de los mismos que constituyen un foro para intercambio de ideas y al mismo tiempo un estimulo para la investigación. Así nació en 1977 la Sociedad Ibérica de Biomecánica cuya alma ha sido desde el primer momento nuestro Secretario, el Dr. Mariano FERNANDEZ FAIREN, que ha sido el principal artífice de los avances que lentamente ha ido adquiriendo nuestra Sociedad. En las Tablas I nombramos los Socios Fundadores y los Presidentes de la misma. Como muestra de la labor realizada también en la tabla no. II citamos los Congresos y Simposiums organizados.

\section{TESIS DOCTORALES}

A lo largo de los años nos ha tocado dirigir o estar en tribunales de Lectura de Tesis. Hemos tenido ocasión de comprobar el valor de las mismas. La investigación tiene que realizar largos caminos para encontrar hallazgos importantes. Por ello más que descubrimientos espectaculares encontramos en las mismas una perfecta forma que demuestra la capacidad y vocación del doctorado. Como dice Umberto ECO, «La Tesis ha activado el metabolismo intelectual... puede suceder que os percateis de tener una vocación investigadora».

Interesa resaltar el gran número de Tesis Doctorales que directa o indirectamente relacionadas con la Biomecánica, se han presentado últimamente. En la Tabla No IV hemos procurado recoger la mayor parte de

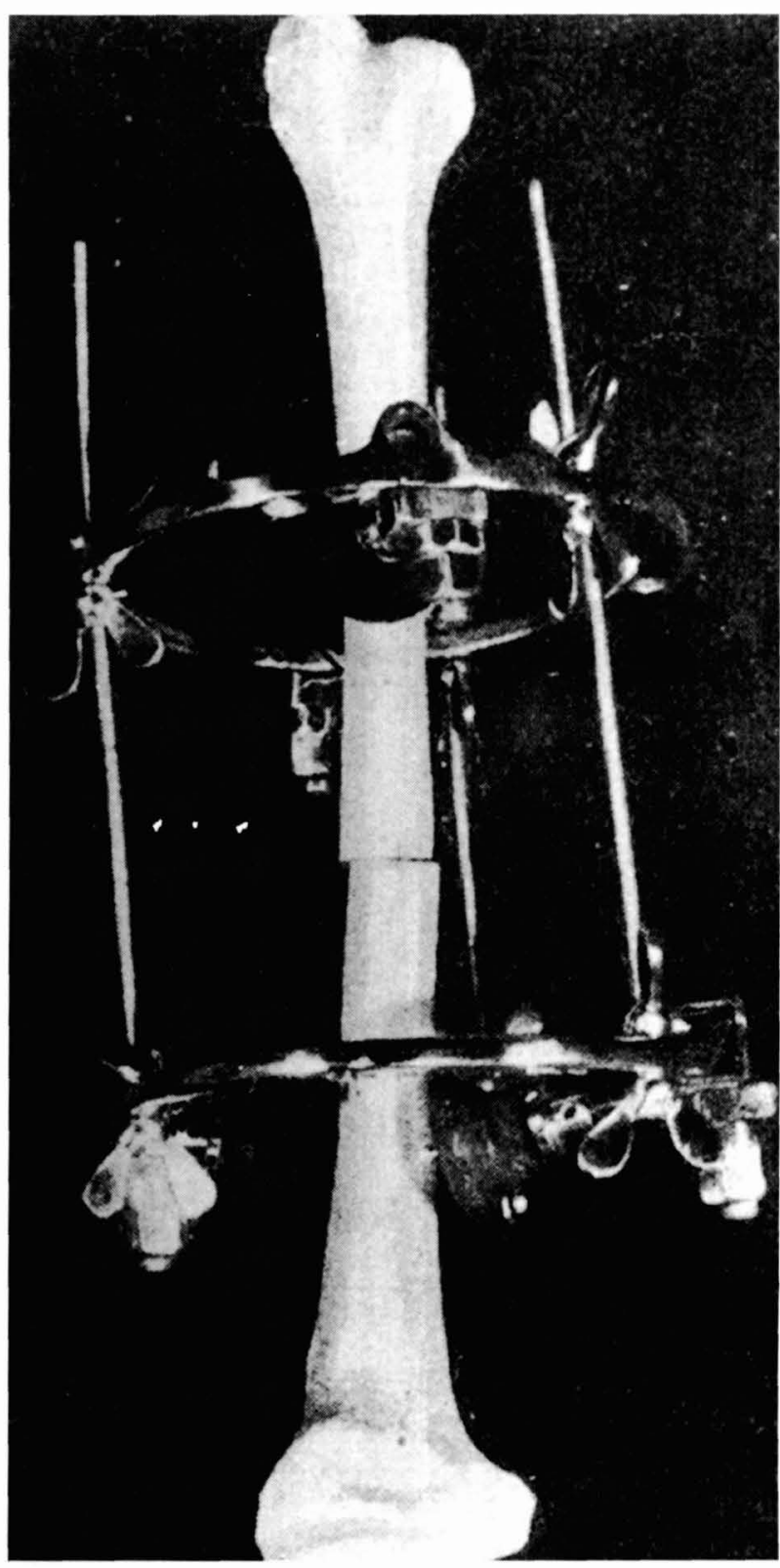

Fig. 8. Fijador externo presentado por el Dr. MENDOZA en 1950 Obsérvese su semejanza con el aparato Ilizarov. 

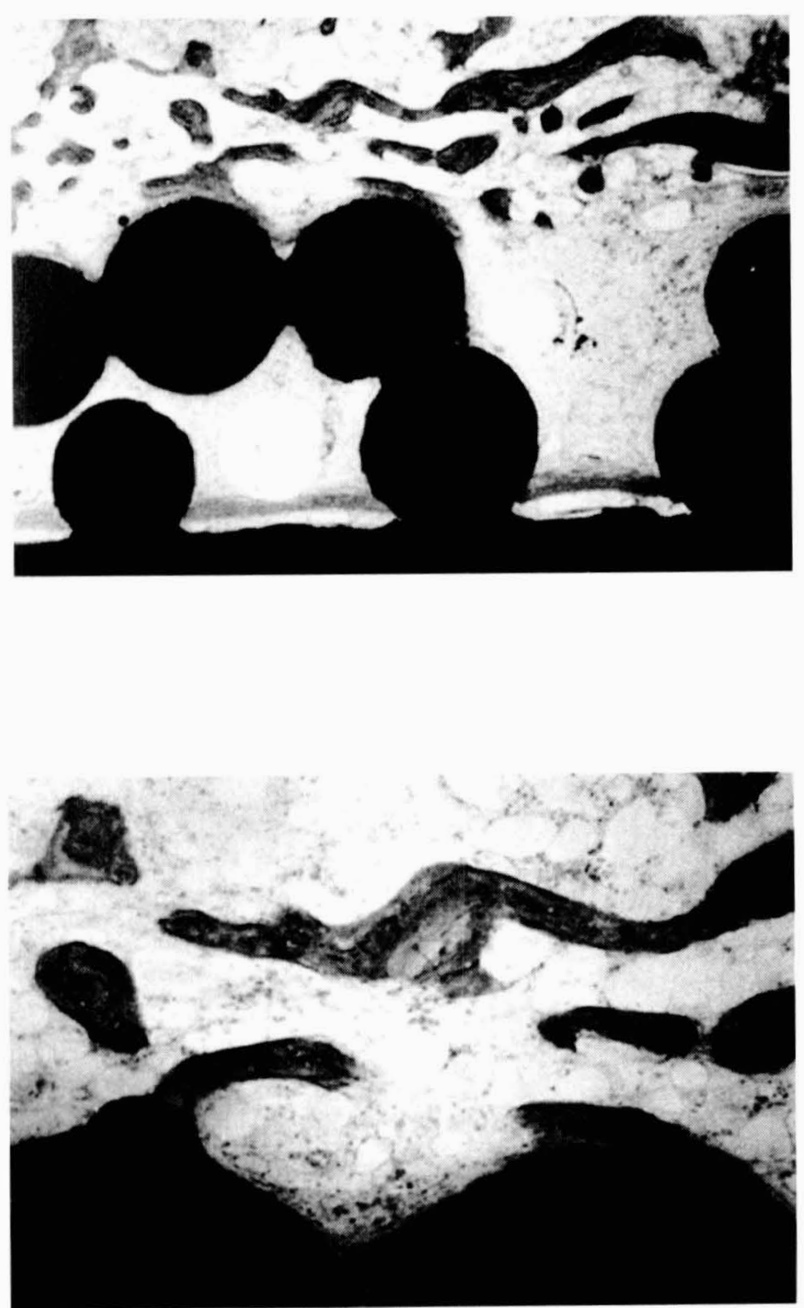

Fig. 9. Fijación de una protesis de cadera (Universidad Politécnicas de Barcelona, Prof. J. PLANELL y Dr. M. FERNANDEZ FAIREN). las mismas. Es lamentable que el gran trabajo que ello representa se pierda. Suelen conocerla a fondo el doctorando y el Director de la Tesis, la leen los miembros del Tribunal pero queda desconocida para el resto del público científico, con la Tabla que adjuntamos pretendemos que se conozcan más las Tesis, y creo que sería una gran labor de la Sociedad poseerlas y ponerlas a disposición de los investigadores. Se evitarían muchas repeticiones y se facilitaría el trabajo.

\section{CONCLUSIONES}

Terminamos con las siguientes peticiones:

1. Solicitar más recursos para que en los Hospitales importantes y en las Universidades Politécnicas haya el correspondiente Laboratorio de Biomecánica.

2. Que las empresas de instrumental quirúrgico realicen investigación propia.

3. Que se crea la Asignatura de Biomecánica en los estudios de segundo grado de las Facultades de Medicina, Biología y Universidad Politécnica.

4. Que continúe el entusiasmo por las Tesis de Biomecánica en las Facultades de Medicina y Universidades Politécnicas, pero que ésta también se extienda a los graduados de la I.N.E.F., cuyos estudios son de grado superior.

5. Unificar esfuerzos de forma que se conozcan lo que se hace en el resto del país y que aumente la colaboración.

Nuestra Sociedad va adquiriendo su madurez, buena prueba de ello es la presente Revista que con tanta ilusión ha preparado nuestro Presidente, Fernando LLANOS. El examen que hemos hecho desde servirnos de estímulo para continuar, a pesar de las dificultades que desgraciadamente se incrementan en estos últimos tiempos. Con ilusión debemos persistir en la investigación biomecánica, no siempre los frutos de la misma se hallan en relación con los medios y sí con el entusiasmo con que se hacen.

\section{TABLA I}

Sociedad Ibérica de Biomecánica 17 Noviembre 1977

\section{Firman los Estatutos de Constitución los Doctores:}

\section{- C. Hernández Ros.}

- J. Olaguibel.

- M. Fernández Fairén.

\section{Socios fundadores}

- Aguilar Cortés, Francisco

- Alcocer Pérez-España

- Asirón Yribarren, Pedro J.

- Carasa Pérez, Ramiro

- Crespo Neches, Antonio

- Fernández Espeve, Francisco

- Farriol Pico, Joaquín

- Lifante Hurtado, José

- Marín Redondo, Mariano

- Olaguibel Moret, Joaquín

- Palacios Barrasus, José

- Pichel Moure, Carlos M.

- Victoria Díaz, José

\section{Presidentes}

Años

$$
\begin{aligned}
& 1.977-78-79 \\
& 1.980-81-82 \\
& 1.983-84-85 \\
& 1.986 \\
& 1.987-88-89 \\
& 1.990-91
\end{aligned}
$$

Dr. C. Hernández Ros Prof. Nieto

Prof. A. Viladot

Prof. L. García-Font

Prof. Pianell

Prof. L.F. Llanos 


\section{TABLA II}

Congresos y Simposiums de la S.I.B.

$\begin{array}{rll}\text { I } & \text { Madrid } & \text { Dr. Hernández Ros } \\ \text { II } & \text { Sevilla } & \text { Dr. Aguilar Cortés } \\ \text { III } & \text { Barcelona } & \text { Dr. Fernández Fairén } \\ \text { IV } & \text { Valencia } & \text { Dr. Nieto } \\ \text { V } & \text { La Coruña } & \text { Dr. C. Pichel } \\ \text { VI } & \text { Zaragoza } & \text { Dr. García Julve } \\ \text { VII } & \text { Tarragona } & \text { Dr. Giné Comá } \\ \text { VIII } & \text { Ibiza } & \text { Dr. Fernández Fairén } \\ \text { IX } & \text { Pamplona } & \text { Dr. Imizcoz } \\ \text { X } & \text { Madrid (INEF) } & \text { Dr. Hernández Vázquez } \\ \text { XI } & \text { Barcelona } & \text { Dr. J.A. Planell } \\ \text { XII } & \text { Granada } & \text { Dr. J.M. Fernández } \\ \text { XIII } & \text { Madrid (Complutense) } \text { Dr. L.F. Llanos } \\ \text { XIV } & \text { Alcalá de Henares } & \text { Dr. Gómez Pellico }\end{array}$

\section{TABLA III}

Estudio Anátomo Funcional del Astrágalo

Juan ROIG PUERTA

Director: R. ARANDES ADAN

Universidad de Barcelona, 1958

Soporte Muscular de la Bóveda Plantar Estática

Luis Fernando LLANOS ALCAZAR

Director: L. GOMEZ OLIVEROS

Universidad Complutense, Madrid, 1975

Estudio de la Marcha Fisiológica en Individuos normales y en portadores de Endoprótesis

Antonio VILADOT VOEGELI

Director: D. RUANO GIL

Universidad de Barcelona, 1978

Análisis Anátomo-Funcional y Estadístico de los Sistemas Estáticos de la Bóveda Plantar del Pie Plano

Mariano NUÑEZ-SAMPER PIZARROSO

Director: L. GOMEZ PELLICO

Universidad Complutense, Madrid, 1978

Concepción de una Prótesis Vertebral

Pedro VERA LUNA

Director: J. NIETO NIETO

Universidad Politécnica de Valencia, 1979
Estudio de las Lesiones producidas por la Inversión del Pie. Zona de Inversión

Rodrigo MIRALLES MARRERO

Director: A. VILADOT PERICE

Inserción Distal del Semimembranoso

Tomás Angel SANZ PELLICO

Director: L. GOMEZ PELICO

Universidad de Alcalá de Henares, 1981

El Ligamento Lateral Interno del Tobillo

Manuel QUILEZ GALINDO

Director: L. GOMEZ PELLICO

Universidad de Extremadura, 1981

Estudio Experimental de la Unión Tendinosa mediante Técnica Aninotica utilizando el Etil-2Cianoacrilato asociado a Implante de Duramadre Fernando LOPEZ PRATS

Director: A. NAVARRO QUILIS

Universidad Autónoma de Barcelona, 1981

Bases Biológicas del Tratamiento de las Fracturas de Extremidad Inferior del Fémur

Francisco ANDRES MARTI

Director: A. NAVARRO QUILIS

Universidad Autónoma de Barcelona, 1981

Aportaciones al Estudio del Pie Cavo Esencial

Pedro CARPINTERO BENITEZ

Director: J.L. LANCHO ALONSO

Universidad de Córdoba, 1982 
Pie Cavo Idiopático. Estudio Experimental Electromiográfico y Antropométrico

J. MARTIN GUINEA

Director: L. GOMEZ OLIVEROS

Universidad Alcalá de Henares, 1982

Análisis Clínico y Biomecánico de la Estática Raquídea en la Lumbalgia crónica basal

Juan Luis MANZANO MERINO

Director: J. BORRACHERO DEL CAMPO

Universidad Complutense, Madrid, 1983

\section{Estudio Cinemático de la Rodilla Humana}

Juan J. VAZQUEZ SOLSONA

Director: A. VIEDMA MARTINEZ

Escuela Técnica Superior de Ingenieros Industriales, Barcelona, 1984

Biometría de la Tibia

Francisco FORRIOL CAMPOS

Director: L. GOMEZ PELLICO

Universidad Alcalá de Henares, 1985

Contribución al Análisis de la Marcha Humana

Juan Víctor HOYOS FUENTES

Director: P. VERA LUNA

Universidad Politécnica de Valencia, 1985

Estudi Esfero-centric del cap femoral en la Coxartrosi

Josep M $M^{\text {a }}$ SALO I ORFILA

Director: A. VILADOT PERICE

Universidad Autónoma de Barcelona, 1985

Estudio de la Marcha en las Neuropatías Hereditarias Sensitivo Motoras

Omaira MOLINA VILORIA

Director: A. VILADOT PERICE

Universidad del Zulia, Maracaibo, Venezuela, 1986

L'Activitat Física i a l'Escola Seguiment Evolutiu

Gustau NADAL RIBERA

Director: J. LLORENS TEROL

Universidad Autónoma de Barcelona, 1986

Estudio Comparativo de la Osteotomía a Cielo abierto y Percutánea en la Elonganción de las Extremidades

Antón ARIEL ARALUCE

Director: J. CAÑADELL CARAFI

Universidad de Navarra, 1986
Análisis cinemático y cinético de la Marcha Humana normal

Miguel GARCIA RAIMUNDO

Director: P. VERA LUNA

Universidad Politécnica de Valencia, 1987

Biometría del Húmero

Manuel GIONONATTI ALIAS

Director: L. GOMEZ PELLICO

Universidad de Alcalá de Henares, 1987

Estudio Experimental sobre diferentes métodos de Osteosíntesis del Raquis Dorso-lumbar

Salvador FUSTER OBREGON

Director: C. PERA

Universidad de Barcelona, 1987

Prótesis de Cadera Autobloqueante de Müller sin Cementar

Antonio-Jesús CARBAYO MARTURET

Director: J.R. VALENTI

Universidad de Navarra, 1987

Estudio Experimental sobre el Tratamiento de los Puentes Oseos Epifisio-metafisarios mediante distracción fisaria

Jesús AZCARATE GANITANO

Director: J. M CAÑADELL CARAFI

Universidad de Navarra, 1987

Acción de las corrientes eléctricas de bajo amperaje en la Regeneración ósea

Xavier MORAITER

Director: A. NAVARRO QUILIS

Universidad Autónoma de Barcelona, 1987

Estudio Biomecánico y Clínico de la Reducción y Redesplazamientos delas Fracturas de Fémur con la Tracción de Neufeld: Conclusiones Terapéuticas José ARANCIL SILVESTRE

Director: A. NAVARRO QUILIS

Universidad Autónoma de Barcelona, 1988

Modificaciones Funcionales de la Articulación Fémoro-rotuliana tras intervenciones quirúrgicas sobre el aparato extensor

Antonio MOLINA ROS

Director: R. BALIUS JULI

Universidad Autónoma de Barcelona, 1988

Biomecánica Femoral

Ramón CASTELEIRO GONZALEZ

Director: L. GOMEZ PELLICO

Universidad de Alcalá de Henares, 1988 
Análisis Biomecánico del movimiento abducción del brazo en el plano frontal

José Luis RODRIGUEZ PEREZ

Director: P. VERA LUNA

Universidad Politécnica de Valencia, 1989

Desarrollo del ángulo de torsión tibio-peroneo durante el crecimiento y su relación con la morfología del Pie

José Antonio PASCUAL MAIQUEZ

Director: L. GOMEZ PELLICO

Universidad de Alcalá de Henares, 1989

Análisis del proceso de reparación ósea mediante sistemas de fijación externa

Jaime PRAT PASTOR

Director: L. GOMEZ PELLICO

Universidad de Alcalá de Henares,1989

Estudio Biomecánico comparativo entre diferentes sistemas de fijación externa

José Antonio JUAN FENOLLOSA

Director: L. GOMEZ PELLICO

Universidad de Alcalá de Henares, 1989

Artrodesis Lumbosacra

Frederic FONT I VILA

Director: A. NAVARRO QUILIS

Universidad Autónoma de Barcelona, 1989

Método de medición de tensiones para plastias de ligamento cruzado anterior.

Análisis experimental con tendón de Aquiles

Rudolf MORGENSTERN

Director: D. RUANO GIL

Facultad de Medicina de Barcelona, 1989

Aspectos Radiográficos y Gammagráficos de la Reacción Osea a la Prótesis no Cementada de Cadera Francisco Javier CABO CABO

Director: A. FERNANDEZ SABATE

Universidad de Barcelona, 1990

Estudio de las presiones fémoro-patelares tras osteotomías de adelgazamiento de rótula

Rafael ARIAZA LOUREDA

Director: F. VAQUERO GONZALEZ

Universidad Complutense de Madrid, 1990

Podometría del radio

Enrique SOLOGAISTUA CENDOYA

Director: L. GOMEZ PELLICO

Universidad de Alcalá de Henares, 1990
Biometría del coxal

Javier FERNANDEZ CAMACHO

Director: L. GOMEZ PELLICO

Universidad de Alcalá de Henares, 1990

Estudio de la sinergia muscular abonista antagonista durante las contracciones isométricas de flexión y extensión del codo

Begoña GAVILANES MIRANDA

Directores: Ch. THEPAUT-MATHIEU, J. ZARRANS

INEF (Vitoria), INSEP (París), 1990

Contribución al Análisis Paramétrico de la Marcha Humana normal. Influencia de la Cadencia, Sexo, Edad, Morfología de la Huella Plantar

Manuel AYORA RODRIGUEZ

Directores: A. VILADOT PERICE, P. VERA

LUNA

Universidad Autónoma de Barcelona, 1990

El nuevo Método de Osteosíntesis destinado a pemitir la carga inmediata en las fracturas trocanteras

Francisco BAIXAULI CASTELLA

Director: A. NAVARRO QUILIS

Universidad Autónoma de Barcelona, 1990

Validez de la corrección desde «H» $a$ «Y» grados en las Osteotomías altas valguizantes de tibia como tratamiento de la gonatrosis fémoro tibial interna Miguel Angel VILATELA FERNANDEZ

Director: A. NAVARRO QUILIS

Universidad Autónoma de Barcelona, 1990

Diseño, creación y ensayo de un extensómetro para la medición de esfuerzos de tracción sobre tejidos conectivos

Rudolf MORGENSTERN

Director: P. ALAVEDRA

Escuela Técnica Superior de Ingenieros Industriales de Barcelona, 1990

Análisis Dinámico de la Marcha. Estudio de los Centros de Presión sobre la Huella Plantar. Influencia de los distintos calzados

$M^{a}$ Amaya SAN GIL SORBET

Director: L. GOMEZ PELLICO

Universidad de Alcalá de Henares, 1991

Análisis de las Prótesis de MITTELMEIER. Experiencia a largo plazo

Angel $M^{\mathrm{a}}$ HIDALGO OVEJERO

Director: J.R. VALENTI NIN

Universidad de Navarra, 1991 
Articulación Talo Calcáneo Navicular. Aportaciones Anatómicas y Experimentales a la Vascularización del Escafoides Tarsiano

Tomás EPELDEGUI TORRE

Director: E. DELGADO BAEZA

Universidad Autónoma de Madrid, 1991

Morfologia i Biomecànica del Peu en el Ballet Nuria MASSO ORTIGOSA

Directores: A. VILADOT PERICE, D. RUANO GIL Universidad de Barcelona, 1991

Análisis de la marcha humana

Concepción DANKLOFF MORA

Director: L. GOMEZ PELLICO

Universidad de Alcalá de Henares, 1991

El enclavado de ENDERS en las fracturas diafisarias de fémur

Joaquín FORES VIÑETA

Director: A. NAVARRO QUILIS

Universidad Autónoma de Barcelona, 1991

Inestabilidad Crónica Anterior de la Rodilla

Manuel MARIN NAVARRO

Director: A. NAVARRO QUILIS

Universidad Autónoma de Barelona, 1991

Influencia Cinética en la Marcha Humana con diferentes tipos de calzado

Olga GALLIFA IRUSO

Directores: A. VILADOT PERICE, R. RAMON

SOLER

Universidad de Barcelona, 1992
Correlación y aptitud morfológica de la mujer en el deporte

Juan Antonio AZCONA TOSCA

Director: R. MORGENSTERN

Facultad de Medicina de Barcelona, 1992

Biomecánica del tarso en el salto

Antonio TURMO GARUZ

Director: R. MORGENSTERN

Facultad de Medicina de Barcelona, 1992

Evaluación de la Fuerza Propulsiva en Natación y su relación con el entrenamiento y la técnica

Raúl ARELLANO COLOMINA

Director: J. VILLA CASTELLAR

INEF, Granada, 1992

Biomecánica de la articulación del codo en el servicio en el tenis

Rosa CASTILLO OMEDES

Director: D. RUANO GIL

Facultad de Medicina de Barcelona, 1992 